\title{
A NEW APPROACH TO THE DIAGNOSIS OF SEPSIS AND PURULENT-INFLAMMATORY DISEASES
}

\author{
Bulavenko O. V., Ostapiuk L. R., Rud V. O.
}

\section{INTRODUCTION}

Sepsis holds the second-highest mortality rate in the world after cardiovascular disease. The problem of sepsis is more than urgent, and in 2017, the World Health Organization (WHO) officially called sepsis a threat to the world and adopted a resolution about this disease. According to the WHO, sepsis is recorded annually in 30 million patients. It leads to 6 million deaths, most of which could be prevented. After all, sepsis is the result of violating the regulation of the response of the macroorganism to infection, manifests with damage to the body's own tissues and organs and concludes with life-threatening multiple organ failure. In the early stages of sepsis, it can be well treated if it is diagnosed early and the effective treatment and monitoring of patients conditions are timely. However, currently the solution of this problem is difficult due to the lack of effective methods of early diagnosis and pathogenetic treatment of this disease ${ }^{1}$.

The 70th WHO Assembly adopted the resolution about the need of developing the clinical guidelines for the prevention of sepsis, its diagnosis and treatment. This issue is to be re-examined by the 73rd WHO Assembly. Therefore, the relevance of this problem is currently very high, but not yet solved, despite the large number of scientific research.

Sepsis during pregnancy and postpartum period remains the leading cause of maternal morbidity and mortality worldwide. Its occurrence is characteristic of both developing and highly developed countries. It should be noted that there are significant difficulties in the diagnosis of obstetric sepsis compared with the diagnosis of sepsis in the general population. First of all, the onset of postpartum purulent-inflammatory diseases (PPPID) is now often accompanied with an atypical aberrant course, characterized by discrepancy between nonspecific common manifestations and the severity of the local pathological process. In addition, during pregnancy, there are characteristic physiological changes that negate the informativeness of

${ }^{1}$ Singer M., Deutschman C.S., Seymour C.W., et al. The Third Internation Consensus Definitions for Sepsis and Septic Shock (Sepsis-3) JAMA. 2016. Vol. 315, № 8. P. 801-810. URL: https://DOI :10.1001/jama.2016.0287. 
certain diagnostic criteria. In particular, tachycardia and tachypnea can occur during physiological pregnancy. Also, in obstetric sepsis, body temperature does not change significantly. The patients of the general population with sepsis have a characteristic disorder of consciousness, but for pregnant women, drowsiness may be characteristic of the physiological course of pregnancy. For the general population, sepsis is characterized by swelling or a positive fluid balance. At the same time, swelling of pregnant women is also a frequent manifestation, which makes this indicator also uninformative for the diagnosis of obstetric sepsis. For the patients with sepsis hyperglycemia is more typical even in the absence of diabetes. In pregnant women, sugar levels should not change normally, but gestational diabetes is also a common phenomenon that can "hide" the clinical picture of sepsis and complicate its diagnosis ${ }^{2}$.

Given the relevance of the problem of sepsis and purulent-inflammatory diseases at present, the aim of this study is to improve the diagnosis and prognosis of the development of purulent-inflammatory diseases and sepsis within the method of fluorescence spectroscopy.

\section{Development of a method for the diagnosis of sepsis in surgical practice}

The pathogenesis of sepsis is quite complex, but it can be described simply as the progression and generalization of "local" infection, which is associated with the uncontrolled multiplication of microorganisms, the release of exotoxins in the foci of infection, combined with a decrease in the body's own protective factors. All these components lead to the formation of multiple organ failure. But in order to develop an effective diagnosis and treatment of this serious disease, it is necessary to understand better the essence of the pathogenesis of this disease so that it can be effectively treated at the stage of appearance.

It is known that albumin in blood serum (BS) performs a number of very important functions in the human's body, including transference and detoxification. The characteristic feature of albumin molecules is their ability to form complexes. It should be noted that in the presence of endogenous intoxication in the human's body, there appear conditions for forming albumin molecules with altered properties. The binding centers of albumin are blocked by the products of the metabolism of bacteria, i.e. toxins. Therefore, these albumin molecules cannot fully perform their

${ }^{2}$ Romanko T.G., Dubrov S.O., Sulimenko O.M., Sulimenko E.M. Obstetric sepsis (clinical lecture). Women's Health. 2019. Vol. 1, № 137. P. 10-27. URL: https://doi 10.15574/HW.2019.137.10. 
functions, including transference and detoxification. This causes increased endogenous intoxication in the patient's body. The most important features is that the "total" albumin concentration remains unchanged. At the same time, the "effective" albumin concentration is significantly reduced and is directly proportional to the severity of the disease ${ }^{3}$. It should be stresses that the diagnostic methods used at the initial phase of our studies do not allow the proper recording of changes in albumin molecules due to endogenous intoxication. Therefore, it was fundamentally important to find a method that could enable the recording of these changes in endogenous intoxication and, thus, the diagnosing of septic conditions. Determining the "effective" concentration of albumin is not available by existing diagnostic methods that are widely used in current clinical practice. Hence, it is important to look for a method to record these changes.

The solving of this problem is possible by using physical research methods, which are highly sensitive, accurate and widely used in modern medical practice.

They include the use of laser correlation spectroscopy, electron paramagnetic resonance and several others. In particular, we have focused on the method of fluorescence spectroscopy (MFS), which is now widely used in experimental physics, as well as in economy and in medical practice.

Luminescent analysis is a method of exploring different objects by observing their luminescence. The method of luminescent analysis can help to study the luminescent characteristics of biological objects both in normal and in various pathological conditions. It is possible to record the intrinsic and probe luminescence of tissues by using specific dyes, as well as the luminescence of biological fluids, including BS, urine and synovial fluid. The phenomenon of luminescence becomes clear when one considers that the molecules in the materials under study may ramain in certain discrete energy states. In this case, the transitions of electrons in them from lower energy states will form the absorption spectra of molecules, and their back transitions from the upper excited states will be accompanied by radiation, which is called luminescence. Luminescence is the result of the absorption of light by the system under study and is caused by the transition of its molecules from the energized state to the basic state.

According to the afterglow duration, $\tau$ luminescence is divided into two types:

- fluorescence if $\tau<10^{-7}$ seconds, i.e. the extinction of luminescence occurs very quickly (for the eye, instantly);

${ }^{3}$ Grizunov Y.A., Dobretsov G.E. Serum Albumin in Clinical Medicine. Moscow : Geotar, 1998. 440 p. 
- phosphorescence if $\tau>10^{-4}$ seconds (in this case, the extinction occurs relatively slowly and is often clearly visible to the naked eye).

MFS is now successfully used in the medical practice for conducting upto-date prospective studies based on the latest developments in molecular biology. They allow identifying certain genetic mutations in humans and their individual predisposition to the development of certain pathological conditions. The results of these studies open the way to the successful development of "personalized medicine". It means that it is possible to identify the individual risk of certain diseases for each individual and appropriate measures to prevent the possibility of their occurrence.

In our study, we will thoroughly inverstigate the partial case of luminescence: radiation with short attenuation times $\left(10^{-9}-10^{-7}\right.$ seconds), i.e. fluorescence. We used MFS to diagnose purulent-inflammatory diseases and sepsis.

Fluorescence emission spectra (FS) were studied. They discover the dependence between the fluorescence intensity and the wavelength of the radiation. FS reflect the structure of the energy levels which are the basis for luminescence.

The study took place between January, 2001 and December, 2019. The clinical research centre for this particular investigation was Lviv's municipal clinical hospital of emergency medical services. The luminescent laboratory of the Department of Experimental Physics, at the Ivan Franko National University of Lviv was an experimental research center. The methods of investigation are clinical, laboratory, biochemical, instrumental. The standard diagnostic algorithm was supplemented by the use of fluorescence spectroscopy ${ }^{4}$. The studies were supported by using the MDR-2 and MDR-12 optical monochromators. The excitation of serum was performed in the region of $280 \mathrm{~nm}$, which corresponds to the field of excitation in human serum albumin.

In this section, we shall describe the results of the first phase of the research. The control group in this study was 40 healthy person without chronic diseases, and the main cohort of patients consisted of 100 patients. Among them, there were 15 patients with sepsis, 10 patients with burn injury, 45 patients with surgical inflammatory diseases and 30 patients with aseptic pathology. FS of BS look like a $\lambda$-type curves with maximum fluorescence in the region of 330.1-335.1 nm. The main indicators used for the analysis in the conducted work are the values of fluorescence intensity $I_{F}$

${ }^{4}$ Gerych I., Bulavenko O., Ostapiuk L. Spectral-fluorescent properties of serum as a reliable marker for early diagnosis of sepsis. Journal of Gynecology and Obstetrics. 2014. Vol. 2, № 5. P. 71-74. URL: https://DOI :10.11648/j.jgo.20140205.11. 
and position of $\lambda_{\max }$. This spectrum corresponds to the normal luminescence of human albumin.

In the blood of patients with sepsis, there are two types of albumin molecules: one is normal, and the other is with altered physicochemical characteristics due to the blocking of albumin centers by toxins which are products of bacterial metabolism. As the amount of complete albumin in the BS decreases, the intensity of the FS decreases. At a certain concentration of "pathological" type of albumin with altered physico-chemical characteristics, the pathological "septic" peak is formed. The appearance of the peak is an unfavorable prognostic sign ${ }^{5}$.

Fig. 1 summarizes the results of the study of FS of the BS of donor and patient (aged 33) with sepsis. At the time of hospitalization, the patient was in a critically difficult condition and suffered from verified bacteremia (blood seeding at the time of hospitalization (Dec. 12): Staphylococcus aureus). Fig. 1 shows that the maximum of the fluorescence band of the patient's BS is shifted to the long-wavelength region by $\Delta \lambda=40 \mathrm{~nm}$ (curve 1) relative to the donor's fluorescence band, and the fluorescence intensity $\left(\mathrm{I}_{\mathrm{F}}\right)$ was 0.3 related unites (r.u.) from the donor's $\mathrm{I}_{\mathrm{F}}$. After surgical intervention and the elimination of the source of infection and intensive antiseptic therapy and prolonged bacteremia, the significant improvement and stabilization of the patient's condition were achieved: the analysis of the FS of the patient on the seventh postoperative day revealed that the shift of her band of fluorescence was significantly reduced $(\Delta \lambda=7 \mathrm{~nm})$ (Fig. 1, curve 2$)$.

At the same time, the intensity of this band increased significantly and quite unexpectedly up to $1.07 \mathrm{I}_{\mathrm{F}}$. Due to the subcompensated changes in the absolute quantitative and qualitative content of BS proteins at the time of the examination (biochemical studies showed total protein and protein fractions at the lower limit of normal), the rapid increase in the fluorescence band of the patient's BS cannot be interpreted by absolute hypoproteinemia, which is inherent in the fluorescence of proteins. The only possible explanation for the above phenomenon of increase in the fluorescence band of the patient's BS fluorescence may be the presence of daily pre-infusion 8-10 liters during this treatment period.

Under these circumstances, a regular increase in the fluid component of the BS leads to pseudogipoproteinemia, i.e. a laboratory phenomenon that does not manifest with standart biuret reaction and can only be differentiated from true hypoproteinemia by a special Phillips and van Slyke technique.

${ }^{5}$ Ostapiuk L. Diagnostic and Therapeutic Model of Sepsis and Purulent-Inflammatory Diseases. International Journal of Clinical Medicine. 2019. Vol. 10. P. 577-595. URL: https://doi.org/10.4236/ijcm.2019.1011047. 


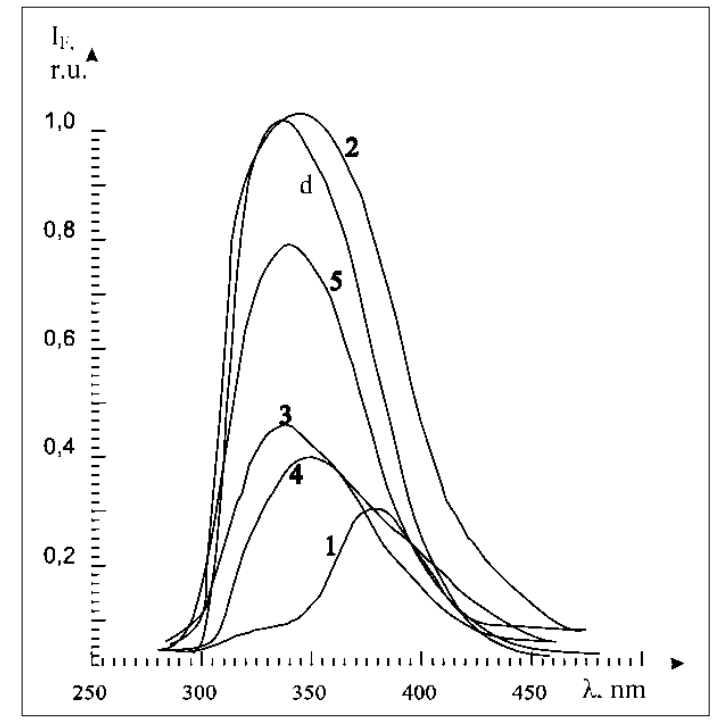

Fig. 1. Fluorescence spectra of blood serum of septic patient:

1 - Dec. 28 ; 2 - Jan. 4; 3 - Feb. 12; 4 - Mar, 19; 5 - June 4 and donor of

BS (340 nm - "normal peak", 380nm - "septic peak")

In our opinion, the forced excess therapeutic dilution of blood during this period caused the quenching of the fluorescence of the BS of the patient and led to the increase of the intensity of the fluorescence bands of her BS. Undoubtedly, the decrease of septic symptoms had a significant impact on the increase of the fluorescence band intensity of the BS (curve 2). Our in vitro studies of the spectral-fluorescence characteristics of standard dilutions of the donor BS with distilled water (DW) (see Fig. 2) confirmed the correctness of the explanation of the reported phenomenon of the increase in the fluorescence band intensity of the BS of this patient (Fig. 1, curve 2).

Besides, the decrease in the content of BS in the samples after the addition of distilled water also led to a significant increase in the intensity of fluorescence bands. The additional confirmation of this assumption was the fact that after a change in the mode of infusion therapy associated with the reduction of septic symptoms and the dominance of sepsis of cardiovascular disorders, which is regular for this stage during the absence of significant changes in laboratory biochemical parameters, there was observed a decrease in the fluorescence intensity of the subsequent blood sampling up to $0,39 \mathrm{I}_{\mathrm{F}}$, which, however, exceeded the initial level $\mathrm{I}_{\mathrm{F}}$, r.u. (Fig. 1, curves 3, 4 relative to curve 1$)$. 

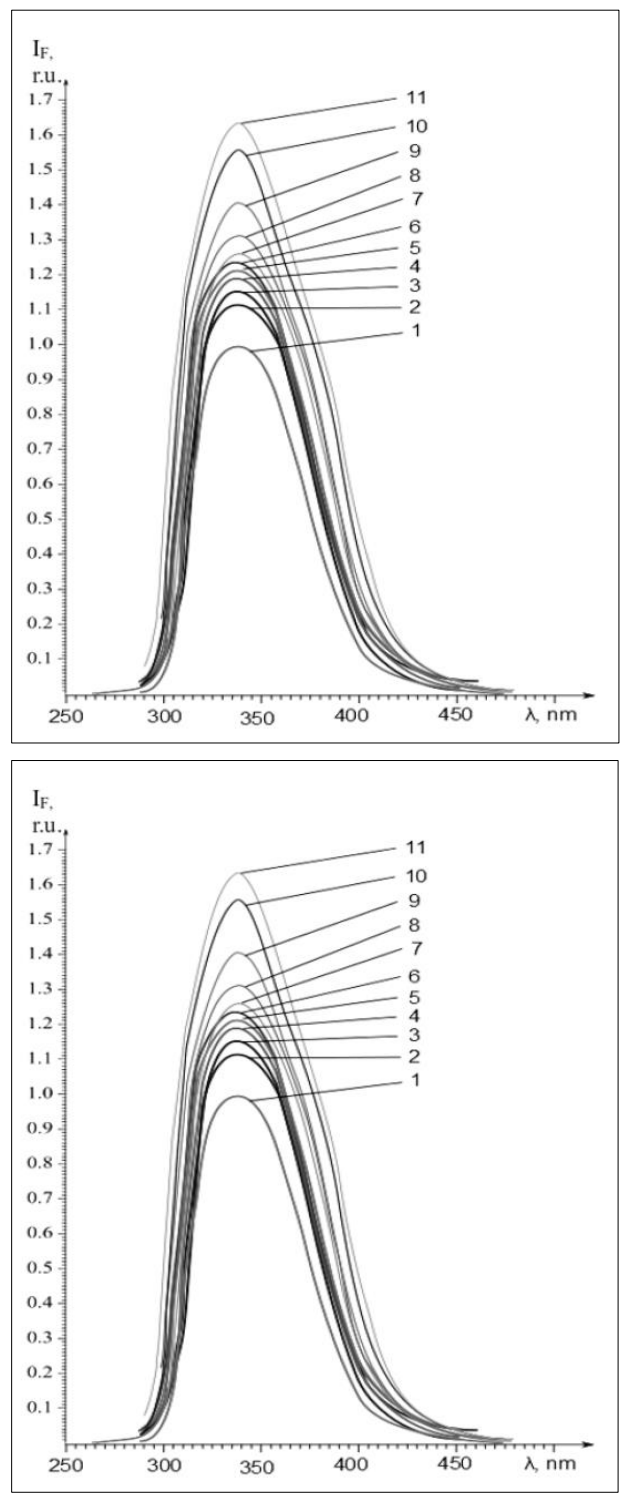

Fig. 2. Effect of dilution with distilled water (DW) on the fluorescence spectra of donor blood serum (BS) (1- BS, $2-90 \% \mathrm{BS}, 3-80 \% \mathrm{BS}$, $4-70 \%$ BS, $5-60 \%$ BS, $6-50 \%$ BS, $7-40 \%$ BS, 8 - $30 \%$ BS, $20 \%$ BS , $10-10 \%$ BS, 11-5\% BS, $12-$ DW : If = 0) 
Later, under the influence of intensive complex therapy, a gradual improvement of the patient's condition with corresponding dynamics of changing spectral-fluorescence characteristics of her BS was observed: the gradual increase of fluorescence band intensity and the reverse shift of its maximum in the spectral region of $337 \mathrm{~nm}$ (Fig. 1, curves 3-5). The significant approximation of the fluorescence parameters of the patient's BS to the corresponding indicators of the donor BS was revealed 2,5 months later after her leaving the hospital. Thus, according to our studies of the BS of this patient, the decrease in the intensity and the shift of the fluorescence band take place due to the presence of an advanced septic process and correlate with the integral indicators of the severity of the clinical condition and bacteremia. The dynamics of changes in the FS of the BS objectively reflects the course of sepsis and correlates with the effectiveness of therapeutic tactics.

The results of another patient with sepsis is depicted in Fig. 3. Due to the well-timed hospitalization and early surgical elimination of the foci of infection, the availability of the septic process was considerably lower. Therefore, at the time of hospitalization, there was only a significant decrease in the intensity of FS of BS, but the "septic" peak was absent, which was a good prognostic sign (Fig. 3).

Analyzing the results in Fig. 3, one can conclude that after eliminating the source of the infection background by an intensive antibiotic therapy, this patient with clinically insignificant course of sepsis during a certain period experienced bacteremia (Klebsiella pneumonia) (curves 1-3).

At this stage of treatment, the decrease in fluorescence band intensity reached maximum $\left(0.16 \mathrm{I}_{\mathrm{F}}\right)$ only at the end of the bacteraemic period. Subsequently, during the gradual recovery of the person treated, there was a significant increase in the fluorescence intensity of the BS up to $0.75 \mathrm{I}_{\mathrm{F}}$ (Fig. 3, curve 5).

Based on the analysis of the results of the study of FS of BS and the clinical features of sepsis in both of the above cases, we have observed the similar dynamics of the disease in the "post-bacterial" period. At the same time, the study of the spectral-fluorescence characteristics of BS of these patients allowed to analyze the nature of the recovery after the disease, unlike the methods provided by the modern standard algorithm for maintainting this category of patients. 


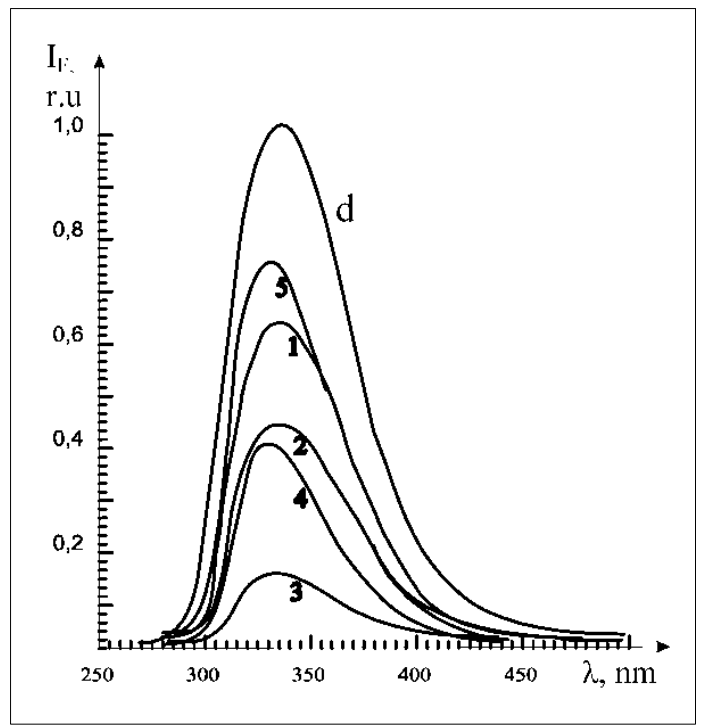

Fig. 3. FS of BS in the patient 2 with sepsis: 1 - June 3; 2 - June 5; 3 - June 6; 4 - June 7; 5 - June 10 and donor of BS. $\lambda_{\mathrm{ex}}=280 \mathrm{~nm}$

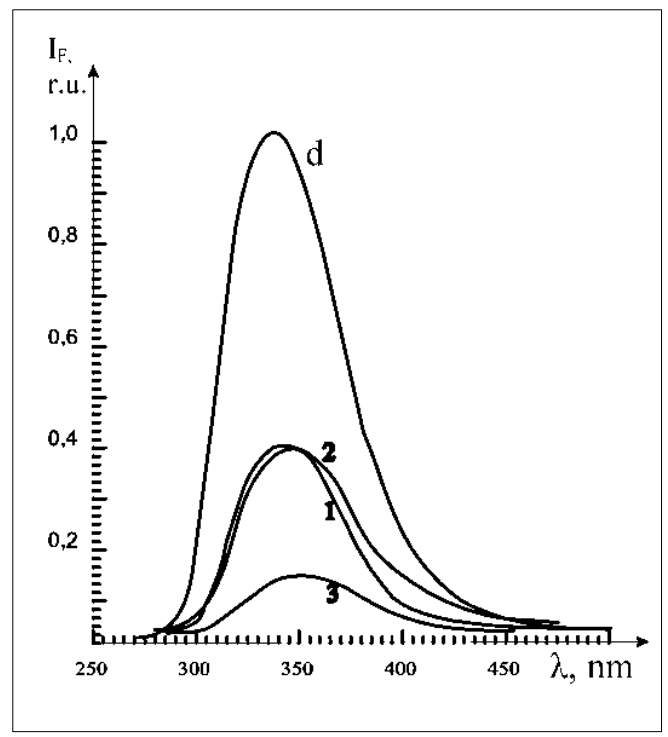

Fig. 4. FS of BS of patient 3 with sepsis and diabetes: 1 - June 3; 2 - June 5; 3 - June 6 and donor BS. $\lambda_{\text {ex }}=280 \mathrm{~nm}$ 
Noteworthy are the results of studies of the spectral-fluorescence characteristics of BS in a patient with sepsis and diabetes (Fig. 4). The patient's condition during the observation period was steadily worsening, despite surgery and intensive antibiotic therapy, which may be well explained by the presence of a number of serious comorbidities and her older age. The negative dynamics of the condition of this patient is reflected by the unfavourable dynamics of the parameters of the spectral-fluorescence characteristics of her BS: a constant decrease in the intensity of fluorescence bands (Fig. 4, curves 1,2,3). The patient died as a result of an advanced process of generalizing infection and multiple organ failure.

It should be noted that patients with diabetes have an increased level of glycolization of albumin of BS. The level of this indicator is $6 \%$ in healthy persons and $9 \%$ in patients with diabetes. Therefore, the albumin molecules in patients with diabetes have reduced amount of free binding centers, which impairs detoxification in these patients and leads to the prolonged purulentseptic state. This is a clear indication of this fact. The presence of the older age and a number of comorbidities further aggravated the prognosis, leading to the development of exitus letalis.

The above results indicate the three most likely scenarios for sepsis. The obtained results indicate that the dynamics of changes in the spectralfluorescence characteristics of BS in patients with sepsis objectively reflect the clinical features of the disease. MFS allows us to use effective therapeutic tactics and to correct it depending on the change of the patient's condition. At the first stage of the disease, the intensity of fluorescence of the BS significantly reduces, which is associated with an increase of the number of defective albumin molecules in the BS, blocked by toxins. Therefore, an important element of therapeutic tactics for purulentinflammatory diseases, including sepsis, is the infusion of $20 \%$ solution of albumin, which allows to replenish the amount of complete albumin in the patient's blood. MFS is also an effective tool for monitoring and controlling the condition of patients with purulent inflammatory diseases and sepsis during treatment.

In the study of the spectral-fluorescence characteristics of the blood serum of patients with purulent-septic complications, two plausible trends were observed: the shift of the fluorescence band maxima for patients with preseptic pathology and sepsis to the long-wave region and decrease in fluorescence intensity up to $70-80 \%$ compared to the donor unit. Both indicators had no correlation with the standard laboratory-biochemical parameters of conventional control of these patients, but correlated properly with the integrated clinical criteria for the severity of the patient's condition and the phenomenon of verified bacteremia. It should be noted that the 
revealed changes in the spectral-fluorescence characteristics of BS in patients with sepsis in most cases were preliminary: they were usually recorded 24-48 hours before the appearance of obvious clinical and laboratory signs of a significant change in the general somatic status of patients (the patent of Ukraine No. 76953) ${ }^{6}$. At the same time, the structure of the excitation spectra of the fluorescence of donors and patients with sepsis is generally similar, but in patients intensities of the excitation spectra are much lower than that in donors.

\section{Optimization of the diagnosis of postpartum purulent-inflammatory diseases}

Postpartum purulent-inflammatory diseases are extremely topical and remain an acute problem of today's obstetrics and gynecology. They are one of the main causes of maternal mortality along with the late gestosis of pregnant women, bleeding and extragenital pathology. The high frequency of PPPID (postpartum purulent-inflammatory diseases) seems a paradoxical problem in the early 21st century, in the period of high achievements in medical science. To some extent, the tendency to develop postpartum complications is connected with the presence of physiological immunodeficiency during pregnancy. The female body during pregnancy and childbirth can be affected by a number of provocative factors that may cause the development of these complications. The widespread prevalence of erased and atypical forms of PPPID makes it difficult to carry out the effective diagnosis and prescribe appropriate effective treatment. The negative impact of PPPID on the subsequent reproductive function of women is also evident that is an important medical and social problem.

Many modern studies in obstetrics focus on the problem of optimizing antibiotic prophylaxis and antibiotic therapy in patients with postpartum purulent-inflammatory complications. At the same time, reliable methods of diagnosis and treatment have not been developed on the basis of the pathogenetically proven concept of sepsis.

The only method for verifying the diagnosis of postpartum endometritis (PPE) is the histological examination of endometrial manual vacuumaspiration of the uterine cavity of postpartum women that discovers inflammatory lymphatic leukocyte infiltration after the 9th day of

\footnotetext{
${ }^{6}$ Method for Early Diagnosis of Septic Complications by the Method of Fluorescence Spectroscopy. Herych I.D., Bulavenko O.V., Ostapiuk L.R., Voloshinovskii A.S. and Myagkota S.V. Pat. № 76953 Ukraine A61B 17/00 G01N. 33/48, G01N 21/64 ; Applicant and Patentee: Pirogov Vinnytsia National Medical University. № 201207441; stat. 19.06.2012; publ. 25.01.2013, Bull. № 2.
} 
postpartum period. When performing the comparative evaluation of various methods of clinical and laboratory diagnostics of PPE, hysteroscopy, determination of acid-base equilibrium and gases in uterine secretions, ultrasonographic examination of the pelvic organs and bacteriological examination of metroaspirite are the most effective ones at present.

Of course, the pelvic ultrasound is a simple method that can be used as a screening and can be one of the initial instrumental methods of examining women after childbirth when the developing of inflammatory process in the postpartum period is suspected. But the possibility of using this method should not be overestimated, as in 50-63\% of cases, ultrasonic signs of endometritis are absent. A vast majority of women leave the maternity hospital on the 3rd or 4th day after childbirth, when there are no visible manifestations of PPPID.

Hysteroscopy is a more accurate method of diagnosing PPE, but it is an invasive, costly method that requires pre-analgesia and is not available in many healthcare institutions. That is why this method can not be widely used for screening in all women after childbirth.

In order to obtain the results of acid-base equilibrium and carry out the bacteriological examination of aspirate in the postpartum period, it is necessary to perform a vacuum aspiration of the uterine cavity in advance to obtain the necessary material. At the same time, like in the case of hysteroscopy, it is necessary to carry out invasive manipulations which cause additional stress in women after childbirth.

The determination of intrauterine pressure, temperature, $\mathrm{pH}$ of uterine contents, proteins of the acute phase of inflammation, IL-1, procalcitonin, histamine, as well as the level of concentration of endotoxins can give valuable information about the presence of inflammatory process in the body of women in postpartum period. At the same time, these methods of diagnosis are quite expensive and are not widely available in most medical institutions. Also, they do not allow to determine accurately the location of the source of the infection. This is especially important in cases where women have extragenital pathologies and other sources of infection in the body. Therefore, these research methods do not allow to differentiate the source of infection in the body.

The use of laser photometry of placenta can optimize the diagnosis of inflammatory changes directly in placenta immediately after childbirth. But it does not provide sufficient information about the status of the uterine cavity in the postpartum period.

Also, prospectful is a method for evaluating the endogenous intoxication syndrome which is based on determining the effective and total concentration of albumin (EAC and TAC) in patients with pelvic 
inflammatory diseases using MFS. It is based on the clear understanding of the physiological processes occurring in the human organism, in particular in the molecules of human serum albumin. As a result, it provides an opportunity to evaluate the prognosis of the disease and the severity of endogenous intoxication in humans.

Within the framework of our study, the standard scheme of examination was extended to include the diagnostics of postpartum endometritis, so we used MFS (the patent of Ukraine No. 133472) ${ }^{7}$. The clinical research centre for this particular investigation was Gynecological department No. 2 of Vinnytsia's City Clinical Hospital No. 2. The luminescent laboratory of the department of experimental physics at the Ivan Franko National University of Lviv was an experimental research centre. The overall research was carried out from 2014 to 2018. The control group constituted 40 new mothers with uncomplicated course of the postpartum period. The main cohort of patients consisted of 170 new mothers with postpartum purulent-inflammatory diseases.

Women with single pregnancy, who had histologically confirmed diagnosis of PPPID in the postpartum period, were involved in the research. These women were informed beforehand and gave an informed consent for participation in this study. Postpartum period after multiple pregnancies, period after antenatal death of the fetus, decompensated somatic illnesses, presence of primary immunodeficiency among postpartum women, presence of HIV infection, tuberculosis (pulmonary and extra-pulmonary), diabetes and a presence of oncological pathology were all considered as exclusion criteria.

The methods of investigation were clinical, laboratory, biochemical, instrumental (uterus and ovarian sonography, bacteriologic and histologic analysis of uterine aspirate, fluorescent spectroscopy), mathematical and statistical (logistic regression and ROC-analysis).

The value of fluorescence intensity of BS in a healthy person is $0.86-1.00$ r.u. If the intensity is more than 1 r.u., it may be connected with increase of circulating blood volume during pregnancy or due to the effects of infusion therapy. An increase of this indicator is not a prognostically adverse sign. Reducing $\mathrm{I}_{\mathrm{F}}$ to 0.8 and below indicates that there is a certain risk of purulent-inflammatory diseases. But this complication may not exactly develop - it is related to the presence of premorbid background and immune characteristics in patients' organisms. The position of the maximum

\footnotetext{
${ }^{7}$ Method of Early Diagnosis of Postpartum Purulent-Septic Complications Using the Method of Fluorescence Spectroscopy. Bulavenko O.V., Ostapiuk L.R., Rud V.O., Voloshinovskii A.S. and Malui T.S. Pat. № 133472 Ukraine GO1N 33/48 (2006.01) GO1N 21/64 (2006.01); Applicant and Patentee: Pirogov Vinnytsia National Medical University. № u2018 10669; stat. 29.10.2018; publ. 10.04.2019, Bull. № 7 .
} 
fluorescence bands in healthy people is within $330.1-335.1 \mathrm{~nm}$. Their offsets into the shortwave region (less than $330.1 \mathrm{~nm}$ ) are not a prognostically adverse signs. However, shifts to the longwave area indicate the risk of purulent-inflammatory diseases. It should be noted that in older patient there also is a gradual smooth wavelength shift of the serum fluorescence spectra.

In patients of the control group, no significant shift of $\lambda_{\max }$ was detected (the position of the maximum fluorescence band in healthy people is within 330.1-335.1 nm). In most cases, fluorescence intensity is in the range of $0.81-1.31$ r.u. compared to $\mathrm{I}_{\mathrm{F}}$ of $20 \%$ donor albumin, which spectralfluorescence characteristics were taken from measurements as a standard. The observed increase of $I_{F}$ may be related to the effects of relative hypoproteinemia, which persists in the postpartum period, as well as due to exposure of infusion therapy, presented in Fig. 2. This information is very important in the analysis of the results of our study of the FS of BS of women in the main cohort. This section will present some of the most interesting results. Some results of the study of FS of BS of patients of control group are depicted in Fig. 5 and Fig. 6 . The average $I_{F}$ in the patients of this group is 0.96 , and the average $\lambda_{\max }$ in this group is $333 \mathrm{~nm}^{8}$.

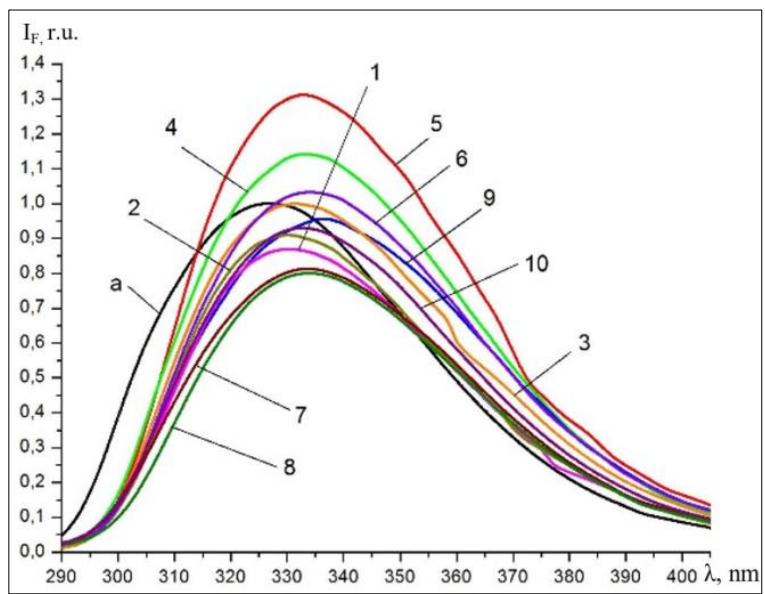

Fig. 5. Fluorescence spectra of serum of healthy women after childbirth in the patient of a control group with uncomplicated postpartum period (1-10) and $20 \%$ solution of donor albumin (a) $\left(\lambda_{\mathrm{ex}}=280 \mathrm{~nm}\right)$

${ }^{8}$ Justification of the feasibility of using the fluorescence spectroscopy method in the complex diagnosis of postpartum endometritis / O. Bulavenko et al. Women's Health. 2016. Vol. 3. P. 71-75. 


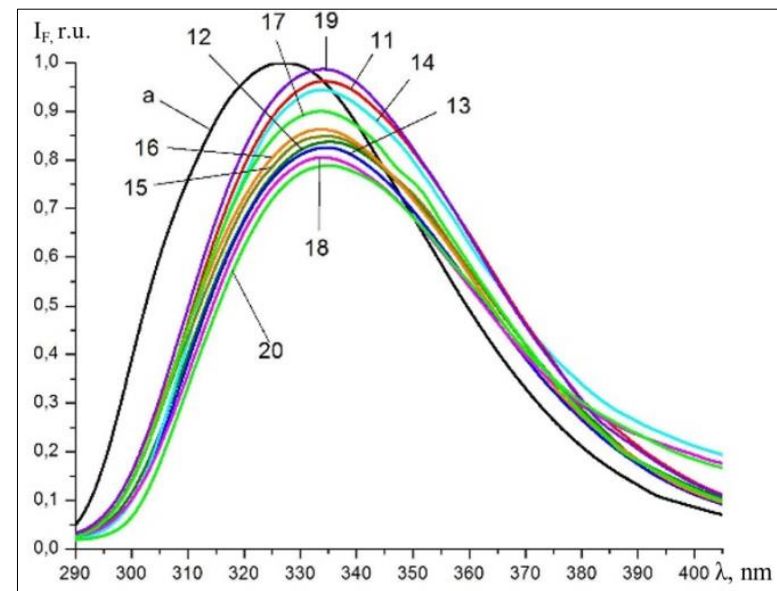

Fig. 6. Fluorescence spectra of serum of healthy women after childbirth in the patient of a control group with uncomplicated postpartum period (11-20) and $20 \%$ solution of donor albumin (a) $\left(\lambda_{\mathrm{ex}}=280 \mathrm{~nm}\right)$

Now we shall describe the number of results of spectral-fluorescence characteristics of BS in women with PPPID, which are presented in fig. 7-10. In these figures, for the sake of comparing, there are also depicted some results for a sepsis patient (see Fig. 1). These characteristics are integral indicators that reflect the balance between the processes of bacteremia and the impact of treatment interventions, including infusion therapy.

Particularly important is the study of the FS of the BS of patients in dynamics during treatment. They reproduce changes in the spectralfluorescence characteristics.

Quite interesting are the results of the study of the FS of the BS of the patient 60 after labour, depicted in Fig. 7. She was hospitalized in the gynaecological department of Vinnytsia's city clinical hospital at the $13^{\text {th }}$ day after the childbirth. This patient had a gynaecological disease (cervix erosion), and an episiotomy was performed during the labour. In the postpartum period, mild anaemia and 3rd-degree vaginal cleanliness were revealed. The ultrasound diagnostics also showed an anomaly of uterine development with enlarged cavity with hyperechogenic content. Three blood samples were taken to see the dynamics. The fluorescence intensity of BS was slightly decreased for the second sample (curve 60'). She had some complaints (general weakness, fever up to $39.5^{\circ} \mathrm{C}$ ). At that time, the vacuum aspiration of the walls of the uterine cavity was performed for therapeutic purposes. As a result, the patient's condition improved (curve 60"), and subsequently, she left the hospital in a satisfactory condition. 
The importance of the study of FS of BS of patient 61 after childbirth in the dynamics is depicted in Fig. 8. This patient was treated for mycoplasmosis and extragenital pathology (chronic bronchitis). There was the threat of a premature childbirth at the $32^{\text {nd }}$ week of her pregnancy. There was a 1st-degree rupture of the cervix during the delivery.

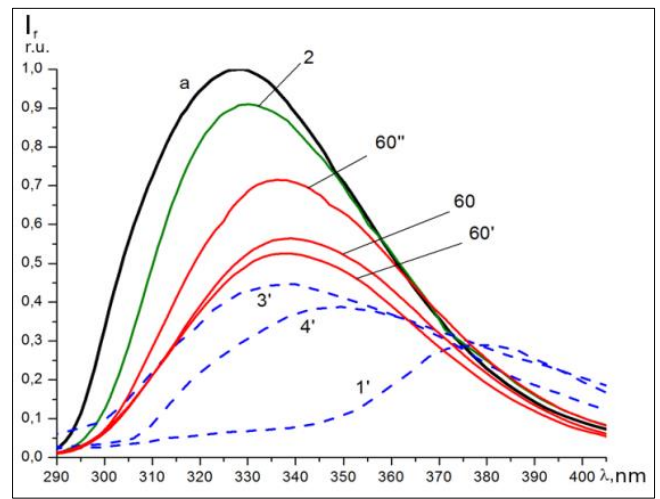

Fig. 7. Fluorescence spectra of blood serum in patient with postpartum endometritis in dynamics $\left(60-24.02 .2015 ; 60 '-26.02 .2015,60{ }^{\prime} '\right.$ '

29.02.2015), women with uncomplicated course of postpartum period (2), patient with sepsis $\left(1^{\prime}, 3^{\prime}, 4^{\prime}\right)$ (see Fig. 1) and $20 \%$ donor albumin (a) $\left(\lambda_{\mathrm{ex}}=280 \mathrm{~nm}\right)$

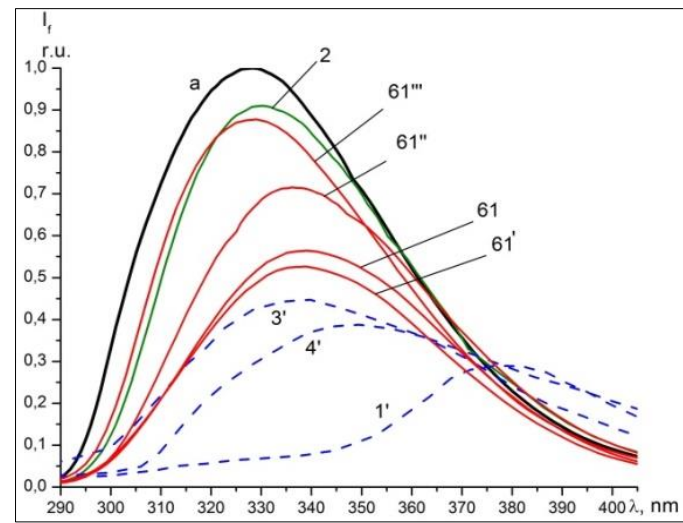

Fig. 8. Fluorescence spectra of blood serum in patient with postpartum endometritis in dynamics $\left(61-2.02 .2015 ; 61 '-4.02 .2015,611^{\prime} \prime-6.02 .2015\right.$, $61 '$ ' ' - 30.04.2015), women with uncomplicated course of postpartum period (2), patient with sepsis $\left(1^{\prime}, 3^{\prime}, 4^{\prime}\right)$ (see Fig. 1) and $20 \%$ donor albumin (a) $\left(\lambda_{\mathrm{ex}}=280 \mathrm{~nm}\right)$ 
During the analysis of vaginal output, bacterial vaginosis was detected. Complaints, i.e. the lower abdominal pain and fever up to $38^{\circ} \mathrm{C}$ in the patient, appeared on the $23^{\text {rd }}$ day of the postpartum period. Patient 61 was admitted to the gynaecological department on the 24th day of her postpartum period. After the vacuum aspiration of the uterine cavity walls, the endometrial histological study revealed endometritis.

Quite informative are the results of the study of the spectral-fluorescence characteristics of the BS of another woman with endometritis after the childbirth, shown in Fig. 9. She had a complicated somatic anamnesis (transferred pleurisy in 2013, urolithiasis), chronic adnexitis. In childbirth, the anhydrous period duration was 6 hours 30 minutes. In the postpartum period, anemia, proteinuria, 3rd-degree purity of the vagina and the expansion of the uterine cavity according to ultrasound examination were revealed. After the manual vacuum aspiration of the walls of the cavity of the uterus of Patient 62, antibacterial and uterotonic therapy were performed. We investigated the FS of the BS on July $15^{\text {th }}, 2015$, and revealed a significant decrease in the fluorescence intensity to 0.35 r.u. and a noticeable long-wave shift of its band (curve 62). In the following experiment, a marked increase of $I_{F}$ of BS of this patient was recorded up to 0.6 r.u. and the shift into the shortwave region (see curve 62') was fixed.

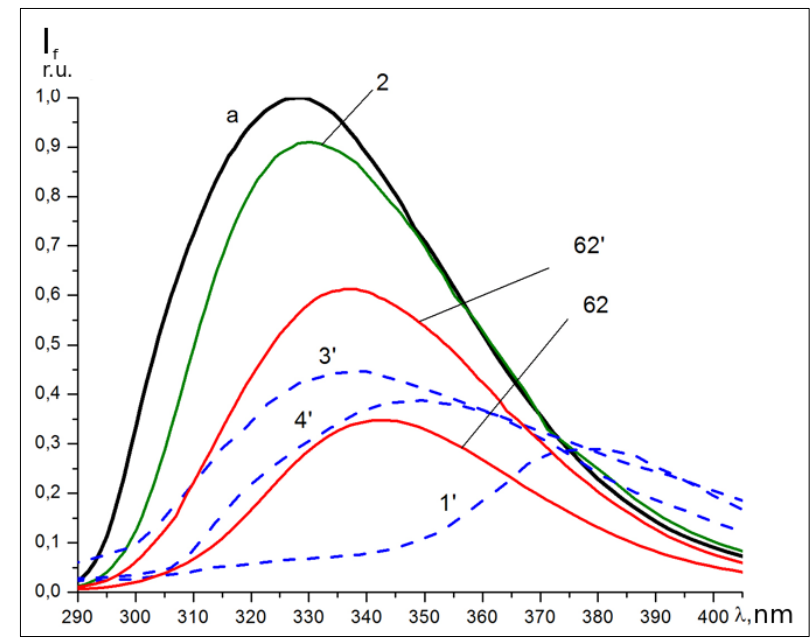

Fig. 9. Fluorescence spectra of serum of a woman after childbirth with endometritis in dynamics $(62-14.02 .2015 ; 62 '-17.02 .2015)$, a woman with uncomplicated course of postpartum period (2), patient with sepsis $\left(1^{\prime}, 3^{\prime}, 4^{\prime}\right)$ (See Fig. 1) and $20 \%$ donor albumin (a) 


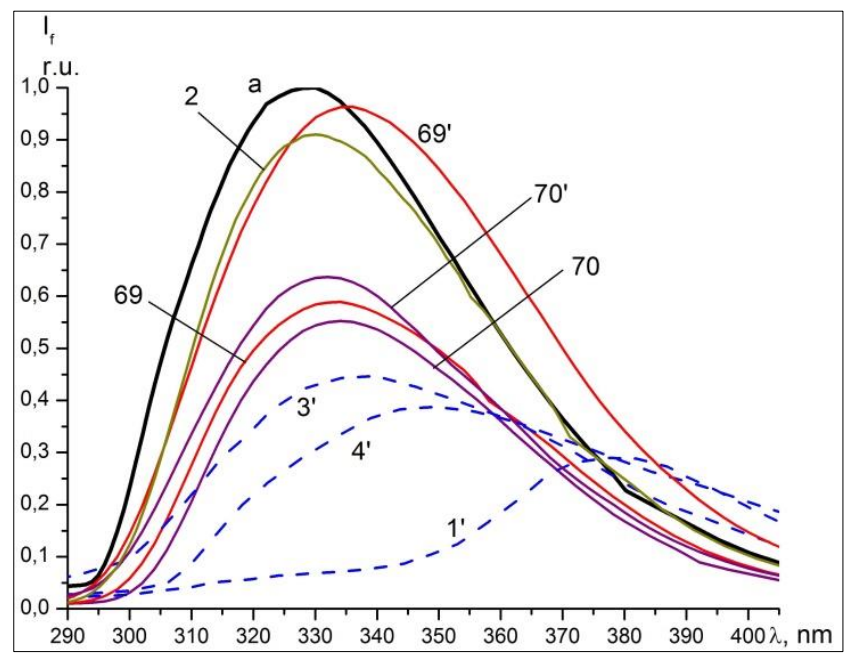

Fig. 10. Fluorescence spectra of serum of women after childbirth with endometritis in dynamics $\left(69-20.02 .2014\right.$ and $69^{\prime}-10.03 .2014$, $70-20.02 .2014$ and $\left.70^{\prime}-24.02 .2014\right)$, woman with uncomplicated course of postpartum period (2), patient with sepsis ( $\left(1^{\prime}, 3^{\prime}, 4^{\prime}\right)$ and $20 \%$ donor albumin (a).

Fig. 10 presents the FS of the BS of two more women with postpartum endometritis. The growth of $I_{F}$ of patient 69 from 0.59 r.u. (curve 69) to 0.96 r.u. (curve 69') correlates with the improvement of her condition during treatment. In the initial study, a considerable decrease of $\mathrm{I}_{\mathrm{F}}$ of the BS (curve 70) of patient 70 was revealed. After the vacuum aspiration on February $20^{\text {th }}$, 2014 and the following treatment, the patient's condition improved significantly. This is confirmed by the results of the study of the FS of the BS (curve 70'). On the basis of detailed information of the spectralfluorescence parameters of BS of patients, it is possible to prescribe effective treatment and to preserve the reproductive health of women after childbirth.

In addition to the scenarios of changes in the spectral-fluorescence characteristics of BS in patients with PPPID, presented in Fig. 7-10, if treatment is ineffective, another scenario can be observed, which is characterized by the decrease of the intensity of fluorescence bands and their shift to the long-wave region. In this case, the appearance of sepsis in patients is not excluded.

It is very important to use albumin infusions, including its significant amount in the treatment of patients with severe forms of purulentinflammatory diseases. It is also recommended to investigate FS of BS in 
dynamics, using MFS. The positive dynamics of changes in the FS of BS in patients indicate the need to complete the process of treatment.

Completing this section, it should be mentioned that in patients with purulent-inflammatory diseases, including sepsis, there are changes in structure of albumin molecules and a decrease in the level of "effective" albumin of blood serum, being capable of performing its functions, including detoxification. Therefore, the pathogenetic component of the treatment of these diseases is antibiotic therapy and infusion therapy with albumin solutions to replenish the amount of albumin in blood serum ${ }^{9}$. According to the latest International Guidelines for the treatment of sepsis and septic shock, experts suggest using albumin in large volumes for infusion therapy.

Thus, in patients with postpartum purulent-inflammatory diseases dicrease in the fluorescence intensity of BS dilutions more than $30 \%$ and a long-wave shift of the $\lambda_{\max }$ more than $10 \mathrm{~nm}$ were found. The decrease in fluorescence intensity and long-wave shift are negative prognostic features and correlate with changes in FS of BS in patients with sepsis (see curves 1', 3', 4' in Fig. 7-10). The spectral-fluorescence characteristics of BS are found to be reliable markers for the diagnosis of purulent-inflammatory diseases in obstetric and gynaecological practice. Their dynamics studies allow to prescribe the effective treatment in time and to prevent the development of obstetric sepsis.

\section{Construction of a prognostic model for the development of postpartum purulent-inflammatory diseases}

In this section we analysed main indicators of 170 new mothers with PPPID (main cohort) and 40 new mothers with uncomplicated postpartum period (control group). These include anamnesis, pregnancy, childbirth, postpartum data and examination results. In the analysis of the obtained results, the presence of a certain sign was marked by the figure " 1 " and its absence by the figure " 0 ". Also, in the study of the BS of patients with the method of fluorescence spectroscopy, the quantitative indicator of the fluorescence intensity of the BS was replaced by a qualitative one, which takes the value " 1 " if the patient had a threshold value $\leq 0,845$ r.u. or " 0 " if the value $>0,845$ r.u. Women were divided into 4 groups by age groups: under 18 years old (Group 0), 18-24 years old (Group 1), 25-34 years old (Group 2) and over 35 years old (Group 3).

\footnotetext{
${ }^{9}$ Optimization of Medical-Diagnostic Approach to Carrying out Vacuum Aspiration at Postpartum Purulent-Inflammatory Diseases / O. Bulavenko et al. Women's Health. 2018. Vol. 7. P. 40-45.
} 
For the sake of isolating the factors, which have a significant effect on the development of PPPID, we applied a stepwise logistic regression method with progressive selection (Forward) ${ }^{10}$.

$$
Q=\frac{1}{1+e^{-R}} * 100 \%
$$

where $\mathrm{e}=2,72 \ldots-$ is the base of a natural logarithm,

$$
R=K+\beta_{1} x_{1}+\beta_{2} x_{2}+\ldots \beta_{n} x_{n},
$$

$\mathrm{K}$ - is a constant; $\mathrm{n}-$ the number of factors which are included in the prognostic mode; $\beta_{\mathrm{i}}$ - coefficients that correspond to a number of calculated factors; $\mathrm{x}_{\mathrm{i}}-$ corresponding numerical values of the factors.

Theoretically, Q can hold a value ranging from $0 \%$ (an impossible event) to $100 \%$ (a constantly occurring event). The meaning of $\beta_{\mathrm{i}}$ coefficients are calculated by the computer program and are represented by the natural log of the correlation of probabilities of corresponding variables. Increasing the value of the independent variable by a unit of measurement would increase the chances of developing complicationsin $\operatorname{EXP}\left(\beta_{\mathrm{i}}\right)$ times.

R-studio V1.1.442 was used to carry out statistical analysis, which was then exported for further analysis in MS Excel. By using logistic regression, we were able to isolate 13 factors, whose effect had the biggest impact on the development of postpartum purulent-inflammatory diseases. The results of calculating regression coefficients of the occurrence of postpartum purulent-inflammatory diseases in the main cohort are depicted in Table 1.

Substituting the obtained $\beta$ i coefficients in (3), and the obtained results in (1), we can determine the likelihood of postpartum purulent-inflammatory diseases:

$$
\begin{gathered}
R=-78,1471+3,8457 * X_{1}+2,5346^{*} X_{2}+1,4007 * X_{3}+2,6075^{*} X_{4}+ \\
+2,6978^{*} X_{5}+4,9746^{*} X_{6}+3,7835^{*} X_{7}+0,2017 * X_{8}+0,2012 * X_{9}+ \\
+6,4036^{*} X_{10}+0,4201 * X_{11}+0,1896^{*} X_{12}+2,7279 * X_{13}
\end{gathered}
$$

In formula (3), the postpartum bedtime (independent variable $\mathrm{X}_{12}$ ) and $\lambda_{\max }$ of BS (independent variable $X_{9}$ ) are quantitative characteristics, age (independent variable $X_{11}$ ) is a factor with four levels from 0 to 3 , where 0 is the age of up to 18 years, $1-18-24$ years, $2-25-34$ years and 3 - over 35 years. The rest of the variables are qualitative dichotomous variables that

${ }^{10}$ A Prognostic Model of the Development of Postpartum Purulent-Inflammatory Diseases / O. Bulavenko et al. International Journal of Clinical Medicine. 2020. Vol. 11. P. 32-42. URL: https://doi.org/10.4236/ijcm.2020.112004. 
take the value " 1 " in the presence of a condition (the presence of surgical treatment in the hospital, TORCH-infection, colpitis or decrease in the fluorescence intensity of blood serum $\leq 0.845$ ) or " 0 " when the condition is absent.

The equation was evaluated according to the Akaike information criterion as well as verified by using $\chi^{2}$ for the likelihood ratio test and by Nagelkerke's R2 (Pseudo R-squared). Furthermore, ROC-analysis was used in order to determine the mathematical credibility of the model and calculate the optimal threshold of decision making (cut-off point).

Table 1

Differences in anamnesis and clinical factors between the control group and main cohort

\begin{tabular}{|c|c|c|c|}
\hline Factor & $\begin{array}{c}\text { Control group } \\
(n=40)\end{array}$ & $\begin{array}{c}\text { Main cohort } \\
\text { (women with } \\
\text { PPPID) } \\
(\mathbf{n}=\mathbf{1 7 0})\end{array}$ & $\mathbf{p}$ \\
\hline Surgery & $12(30 \%)$ & $139(81,8 \%)$ & $<0,001$ \\
\hline Extragenital pathology & $7(17,5 \%)$ & $102(60 \%)$ & $<0,001$ \\
\hline Gynecological diseases & $12(30 \%)$ & $111(65,3 \%)$ & $<0,001$ \\
\hline $\begin{array}{l}\text { Complicated course } \\
\text { of pregnancy }\end{array}$ & $19(47,5 \%)$ & $143(84,1 \%)$ & $<0,001$ \\
\hline $\begin{array}{l}\text { Invasive procedures } \\
\text { during labour }\end{array}$ & $10(25 \%)$ & $89(52,4 \%)$ & 0,002 \\
\hline Risk of miscarriage & $4(10 \%)$ & $40(23,5 \%)$ & 0,058 \\
\hline TORCH-infections & $2(5 \%)$ & $74(43,5 \%)$ & $<0,001$ \\
\hline Colpitis & $7(17,5 \%)$ & $149(87,6 \%)$ & $<0,001$ \\
\hline $\begin{array}{l}\text { Labour duration } \\
>12 \text { hours }\end{array}$ & $2(5 \%)$ & $12(7,1 \%)$ & 0,64 \\
\hline $\begin{array}{l}\text { Vaginal tears during } \\
\text { labour }\end{array}$ & $9(22,5 \%)$ & $69(40,6 \%)$ & 0,03 \\
\hline Labour anomalies & $1(2,5 \%)$ & $61(35,9 \%)$ & $<0,001$ \\
\hline$\lambda_{\max }$ of BS & $333,63 \pm 1,46$ & $335,28 \pm 2,29$ & $<0,001$ \\
\hline $\begin{array}{l}\text { Fluorescence intensity } \\
\leq 0,845 \text { r.u. }\end{array}$ & $6(15 \%)$ & $152(89,4 \%)$ & $<0,001$ \\
\hline Bed days & $4,5 \pm 1,47$ & $5,69 \pm 1,99$ & $<0,001$ \\
\hline Fetal distress & $1(2,5 \%)$ & $36(21,2 \%)$ & 0,005 \\
\hline $\begin{array}{l}\text { Risk of miscarriage and } \\
\text { respiratory disease }\end{array}$ & $2(5 \%)$ & $10(5,9 \%)$ & 0,82 \\
\hline
\end{tabular}


As a result, the ROC-curve was implied in order to demonstrate correlation between specificity and sensitivity. The area under the curve (AUC) was calculated to characterise the model's quality, where the scale varied between 0.5 (method is unacceptable) to $1-100 \%$ which is an indication of the congruence in prognosis based on the model.

The Nagelkerke coefficient of determination demonstrates which part of dispersion of independent variable can be explained by dependent variable introduced to the model and it equals 0,9076. This confirms that the set of variables explains around $90 \%$ of dispersion of dependent variable. The area under the curve (AUC) equals 0,99. The obtained results are depicted in Fig. 11. Determination of the optimal threshold of decision making (cut-off point) was done by calculating optimum cut-off value, which maintains equality between sensitivity and specificity. For this particular model, the threshold was 0,78 , leading to a conclusion that a patient is highly likely to be affected if the risk of occurrence of postpartum purulent-inflammatory diseases is $>0,78$. However, the patient will be unaffected if the calculated risk is $<0,78$.

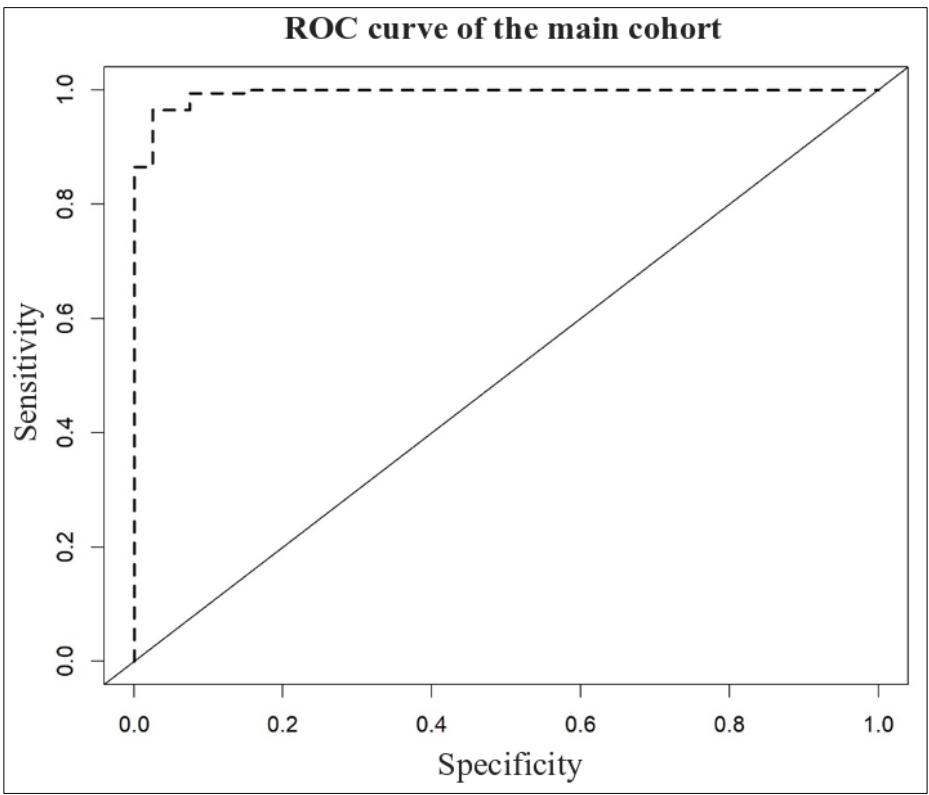

Fig. 11. ROC-curve demonstrates the mathematical model of occurrence of postpartum purulent-inflammatory diseases among the main cohort 


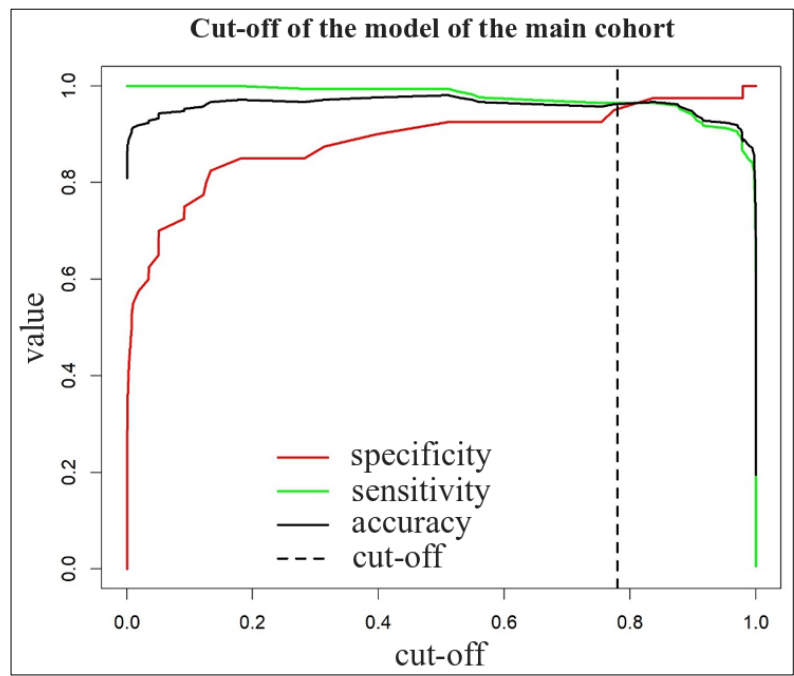

Fig. 12. Threshold of decision making (cut-off point) in relation to specificity, sensitivity and accuracy of the mathematical model for the patients with postpartum purulent-inflammatory diseases in the main cohort

At this threshold value of the decision making (cut-off point), sensitivity is $96,47 \%$, specificity is $97,50 \%$ (Table 2), likelihood ratio of the positive result ( $\mathrm{LR}+$ ) is 38,58 , the likelihood of negative result (LR-) is 0,04 , positive prognostic value (PPV) is $99,39 \%$ while the negative prognostic value (NPV) is $86,67 \%$.

Table 2

Diagnostic value of the mathematical model implied in prognosis of postpartum purulent-inflammatory diseases among new mothers in the main cohort

\begin{tabular}{|l|c|c|c|}
\hline & $\begin{array}{c}\text { Uncomplicated } \\
\text { course of } \\
\text { postpartum } \\
\text { period (n = 40) }\end{array}$ & $\begin{array}{c}\text { Dignosed } \\
\text { postpartum purulent- } \\
\text { inflammatory diseases } \\
(\mathbf{n = 1 7 0 )}\end{array}$ & Total \\
\hline $\begin{array}{l}\text { Calculated } \\
\text { value }<0,78\end{array}$ & $39(97,5 \%)$ & $6(3,53 \%)$ & 45 \\
\hline $\begin{array}{l}\text { Calculated } \\
\text { value } \geq 0,78\end{array}$ & $1(2,5 \%)$ & $164(96,47 \%)$ & 165 \\
\hline Total & 40 & 170 & 210 \\
\hline
\end{tabular}


The obtained model is reliable, with a probability of more than $99 \%$ $\left(\mathrm{p}<0.001 ; \chi^{2}=174.74 ; \mathrm{df}=13\right)$. All 13 factors have been shown to have a provocative effect on the likelihood of postpartum purulent-inflammatory diseases, but their impact is manifested to varying degrees. As noted earlier, EXP $\left(\beta_{\mathrm{i}}\right)$ indicates how many times an increase in the chances of this complication is predicted by increasing the independent variable by one.

For example, the chances of being at risk of postpartum purulent-septic complications with an increase in postpartum staying in the hospital by one day increase in 1,21 times. The most significant influence on the occurrence of postpartum purulent-inflammatory diseases in the main group has the decrease of the serum fluorescence intensity $\leq 0,845$ r.u.: this indicator increases the risk of this complication in 604 times.

Tables 3 and 4 demonstrate selected results of the spectral and fluorescent characteristics of blood serum of both main and control cohorts of patients and corresponding calculation of the probability of development of postpartum purulent-inflammatory diseases. Among the patients in the main cohort (Table 4), only 6 cases had a low likelihood of developing PPPID. If this indicator is below 0,78 (Table 2) for the obtained model, the risk of developing PPPID is low. Such a woman does not require the appointment of special treatment and prevention measures.

Table 3

Probability of PPPID in control group

\begin{tabular}{|c|c|c|c|}
\hline № & $\mathbf{I}_{\mathbf{f}}$ & $\boldsymbol{\lambda}_{\max }$ & Probability \\
\hline 1 & 0,87 & 334,6 & 0,00124 \\
\hline 2 & 0,89 & 335,1 & 0,05022 \\
\hline 3 & 0,99 & 332,9 & 0,00259 \\
\hline 4 & 0,91 & 332,6 & 0,00751 \\
\hline 5 & 1 & 331,1 & 0,09102 \\
\hline 6 & 0,91 & 330,1 & 0,00043 \\
\hline 7 & 0,87 & 330,1 & 0,01822 \\
\hline 8 & 0,99 & 333,1 & 0,00339 \\
\hline 9 & 0,96 & 334,5 & 0,09032 \\
\hline 10 & 0,95 & 335,1 & 0,00056 \\
\hline 11 & 1 & 335,1 & 0,00949 \\
\hline 12 & 0,97 & 333,1 & 0,12568 \\
\hline 13 & 0,92 & 333,1 & 0,00076 \\
\hline 14 & 0,88 & 332,1 & 0,13294 \\
\hline 15 & 0,83 & 335,1 & 0,39702 \\
\hline
\end{tabular}


Probability of PPPID development in the main cohort

\begin{tabular}{|c|c|c|c|}
\hline № & $\mathbf{I}_{\mathbf{f}}$ & $\boldsymbol{\lambda}_{\max }$ & Probability \\
\hline 1 & 0,55 & 334,1 & 0,99989 \\
\hline 2 & 0,58 & 336,1 & 0,99997 \\
\hline 3 & 0,61 & 343,1 & 0,99999 \\
\hline 4 & 0,67 & 336,8 & 0,91405 \\
\hline 5 & 0,77 & 333,3 & 0,95524 \\
\hline 6 & 0,63 & 338,1 & 0,99999 \\
\hline 7 & 0,51 & 337,1 & 0,99999 \\
\hline 8 & 0,53 & 339,1 & 0,99999 \\
\hline 9 & 0,57 & 343 & 0,99912 \\
\hline 10 & 1 & 336,1 & 0,65298 \\
\hline 11 & 0,88 & 333 & 0,526028 \\
\hline 12 & 0,89 & 336,1 & 0,51114 \\
\hline 13 & 0,77 & 332,8 & 0,55993 \\
\hline 14 & 0,73 & 335,1 & 0,180811 \\
\hline 15 & 0,71 & 336 & 0,54868 \\
\hline
\end{tabular}

If this indicator is higher than 0.78 , the patient has a high risk of postpartum purulent-inflammatory diseases. This patient requires a complex of treatment and prevention measures.

\section{CONCLUSIONS}

1. The method of fluorescence spectroscopy makes it possible to carry out diagnostics at the preclinical stage, to assess the threat of critical purulent-septic complications quickly and qualitatively and to monitor the treatment process.

2. Changes in the spectral-fluorescence characteristics of blood serum in patients with sepsis in most cases were preliminary: they were usually recorded 24-48 hours before the appearance of obvious clinical and laboratory signs of a significant change in the general somatic status of patients.

3. In the blood serum of patients with purulent-inflammatory diseases and sepsis, there are structural changes in albumin molecules. This leads to a decrease of the level of "effective" albumin, capable for performing its basic functions, including detoxification and transport. Therefore, the pathogenetic component of the treatment of these diseases is antibiotic therapy and infusion therapy. The aim of using albumin solutions is to replenish the 
amount of "effective" albumin in the blood serum of patients with endogenous intoxication.

4. Designing the prognostic model of the development of postpartum purulent-inflammatory diseases makes possible to identify women with a high risk of developing this pathology and to select an individual diagnostictherapeutic algorithm for each patient according to the modern principles of personal medicine.

\section{SUMMARY}

The problem of sepsis and purulent-inflammatory diseases is very actual now. The unsatisfactory results of treatment of patient with sepsis are largely related to the lack of the effective methods for its early diagnosis. The aim of this research is to improve the diagnosis and prognosis of the development of purulent-inflammatory diseases and sepsis within the method of flourescence spectroscopy. Materials and methods. In the first stage of the study the control group consisted of 40 healthy people without chronic diseases, and the main cohort of patients consisted of 100 patients with purulent-inflammatory diseases and sepsis. At the second stage, the main cohort consisted of 170 women with diagnosed purulent-inflammatory diseases after the childbirth while the control cohort was made of 40 women with an uncomplicated course of postpartum period. Methods of investigation: clinical, laboratory, biochemical, instrumental. The standard diagnostic algorithm was supplemented by the use of fluorescence spectroscopy. The study results were processed with mathematical and statistical methods (logistic regression and ROC-analysis). Results. The study includes three sections. In the first of them, spectro-fluorescence characteristics of blood serum in purulent-septic complications and sepsis in surgical practice were studied using the method of fluorescence spectroscopy. The second section investigates the fluorescence spectra of serum of patients with postpartum purulent-inflammatory diseases. In the third section, a prognostic model for the development of postpartum purulent-inflammatory diseases was designed by using logistic regression and ROC analysis method. Conclusions. In patients with purulentinflammatory diseases, the decrease in the fluorescence intensity of blood serum is observed. In particularly severe cases, there is a significant decrease in the fluorescence intensity and a long-wave shift of the fluorescence spectrum, with a tendency to form a "septic" peak, the appearance of which is an unfavorable prognostic sign. These changes were recorded 24-48 hours before the appearance of obvious clinical and laboratory signs of a significant change in the general somatic status of patients. Spectralfluorescence characteristics of blood serum have been found to be reliable 
markers for the diagnosis of purulent-inflammatory diseases in medical practice, in particular in surgical and obstetric-gynaecological practice. The studies in dynamics makes it possible to prescribe the effective treatment in time and to prevent the development of obstetric sepsis. To estimate the risk of postpartum purulent-inflammatory diseases in women after childbirth, a reliable prognostic mathematical model was designed within the method of logistic regression and ROC analysis with a probability of more than $99 \%$ $\left(\mathrm{p}<0.001 ; \chi^{2}=174.74 ; \mathrm{df}=13\right)$.

\section{REFERENCES}

1. Singer M., Deutschman C.S., Seymour C.W., et al. The Third Internation Consensus Definitions for Sepsis and Septic Shock (Sepsis-3) JAMA. 2016. Vol. 315, № 8. P. 801-810. URL: https://doi:10.1001/ jama.2016.0287.

2. Romanko T.G., Dubrov S.O., Sulimenko O.M., Sulimenko E.M. Obstetric sepsis (clinical lecture). Women's Health. 2019. Vol. 1, № 137. P. 10-27. URL: https:// doi 10.15574/HW.2019.137.10.

3. Grizunov Y.A., Dobretsov G.E. Serum Albumin in Clinical Medicine. Moscow : Geotar, 1998, 440 p.

4. Gerych I., Bulavenko O., Ostapiuk L. Spectral-fluorescent properties of serum as a reliable marker for early diagnosis of sepsis. Journal of Gynecology and Obstetrics. 2014. Vol. 2, № 5. P. 71-74. URL: https://doi:10.11648/j.jgo.20140205.11.

5.Ostapiuk L. Diagnostic and Therapeutic Model of Sepsis and PurulentInflammatory Diseases. International Journal of Clinical Medicine. 2019. Vol. 10. P. 577-595. URL: https://doi.org/10.4236/ijcm.2019. 1011047.

6. Method for Early Diagnosis of Septic Complications by the Method of Fluorescence Spectroscopy. Herych I.D., Bulavenko O.V., Ostapiuk L.R., Voloshinovskii A.S. and Myagkota S.V. Pat. № 76953 Ukraine A61B 17/00 G01N 33/48, G01N 21/64 ; Applicant and Patentee: Pirogov Vinnytsia National Medical University. № 201207441; stat. 19.06. 2012; publ. 25.01.2013, Bull. № 2 .

7. Method of Early Diagnosis of Postpartum Purulent-Septic Complications Using the Method of Fluorescence Spectroscopy. Bulavenko O.V., Ostapiuk L.R., Rud V.O., Voloshinovskii A.S. and Malui T.S. Pat. № 133472 Ukraine GO1N 33/48 (2006.01) GO1N 21/64 (2006.01); Applicant and Patentee: Pirogov Vinnytsia National Medical University. № u2018 10669; stat. 29.10.2018; publ. 10.04.2019, Bull. № 7.

8. Justification of the feasibility of using the fluorescence spectroscopy method in the complex diagnosis of postpartum endometritis / O. Bulavenko et al. Women's Health. 2016. Vol. 3. P. 71-75. 
9. Optimization of Medical-Diagnostic Approach to Carrying out Vacuum Aspiration at Postpartum Purulent-Inflammatory Diseases / O. Bulavenko et al. Women's Health. 2018. Vol. 7. P. 40-45.

10. A Prognostic Model of the Development of Postpartum PurulentInflammatory Diseases / O. Bulavenko et al. International Journal of Clinical Medicine. 2020. Vol. 11. P. 32-42. URL: https://doi.org/10.4236/ ijcm.2020.112004.

\section{Information about the authors:}

Bulavenko O. V.,

Doctor of Medical Sciences, Professor, Head of Department of Obstetrics and Gynaecology № 2 Pirogov Vinnytsia National Medical University 56, Pirogov str., Vinnytsia, 21018, Ukraine

Ostapiuk L. R.,
Candidate of Medical Sciences,
Obstetrician-gynaecologist
Lviv Regional Public Health Centre
45, Lysenka str., Lviv, 79008, Ukraine
Rud V. O.,
Doctor of Medical Sciences,
Professor of Department of Obstetrics and Gynaecology № 2
Pirogov Vinnytsia National Medical University,
56, Pirogov str., Vinnytsia, 21018, Ukraine
Head of the Gynaecological Department № 2
Vinnytsia City Clinical Hospital № 2
20, Zamostianska str., Vinnytsia, 21009, Ukraine

